\title{
The electronically excited states of helium clusters: an unusual example for the presence of Rydberg states in condensed matter
}

\author{
Klaus von Haeften ${ }^{1}$, Tim Laarmann ${ }^{2}$, Hubertus Wabnitz ${ }^{3}$ \\ and Thomas Möller ${ }^{4}$ \\ Hamburger Synchrotronstrahlungslabor HASYLAB at Deutsches Elektronen Synchrotron DESY, \\ Notkestr. 85, 22603 Hamburg, Germany \\ E-mail: Klaus.von.Haeften@rub.de
}

Received 12 August 2004

Published 5 January 2005

Online at stacks.iop.org/JPhysB/38/S373

\begin{abstract}
The nature of the electronically excited states of He clusters and their relaxation mechanisms are investigated by spectroscopy using monochromatized synchrotron radiation. Time correlated fluorescence excitation and energy resolved luminescence spectra of the clusters are recorded in separate wavelength ranges. The size of the clusters and the isotopic constitution is also varied. The spectral features are analysed and discussed particularly with regard to the high lying states and their possible Rydberg nature. While Rydberg states seem not to exist in the interior region of large clusters there is experimental evidence that sharp lines in the spectrum are either due to He Rydberg atoms or excimer molecules in high vibrational states bound at the surface of large clusters or due to very small positively charged clusters with the Rydberg electron outside. The spectra of large ${ }^{3} \mathrm{He}$ clusters exhibit a larger contribution of Rydberg lines than ${ }^{4} \mathrm{He}$ clusters. He clusters also emit fluorescence at energies above the ionization energy of He atoms. This is attributed to the barrier for the injection of electrons into the conduction band which was found to be $1.35 \mathrm{eV}$ for ${ }^{4} \mathrm{He}$ and $0.95 \mathrm{eV}$ for ${ }^{3} \mathrm{He}$ clusters, respectively.
\end{abstract}

\footnotetext{
1 Present address: Lehrstuhl für Phys. Chemie II, Ruhr-Universität Bochum, 44780 Bochum, Germany.

2 Present address: Max-Born-Institute, Max-Born Str. 2a, D-12489 Berlin, Germany.

3 Present address: CEA-SPAM, Centre d'Etudes de Saclay, 91191 Gif sur Yvette, France.

4 Present address: Institut für Atomare Physik und Fachdidaktik Technische Universität Berlin, Hardenbergstr. 36, D-10623 Berlin, Germany.
} 


\section{Introduction}

He clusters are ideal model systems for investigating the evolution of electronically excited states from atoms to the bulk because of their relatively simple electronic structure. Recent investigations have focused on the lowest electronically excited states of He. For bulk liquid He Surko et al (1969) have measured the reflection spectrum in the vacuum ultraviolet (VUV) wavelength range. They identified the two lowest bands A and B as due to perturbed $2 \mathrm{~s}$ and $2 \mathrm{p}$ atomic states. Solid hcp ${ }^{4} \mathrm{He}$ which was irradiated by X-rays by Arms et al (2001) also reveals a molecular-type band in the energy loss spectrum at a similar energy as found for the liquid. Considerable insight into the nature of the electronically excited states of condensed He has been obtained by investigations on clusters by Joppien et al (1993) and von Haeften et al (2001) using monochromatized synchrotron radiation. Owing to the different densities of ${ }^{3} \mathrm{He}$ and ${ }^{4} \mathrm{He}$ clusters and excitation spectra recorded selectively in the visible and near infrared (VIS/IR) and in the VUV spectral ranges it was possible to establish Surko's assignment of band $\mathrm{A}$ and $\mathrm{B}$, and to identify the adjacent bands as due to perturbed $3 \mathrm{~s}, 3 \mathrm{p}$ and $3 \mathrm{~d}$ states. While in the VUV spectrum the $3 \mathrm{~s}$ and $3 \mathrm{~d}$ bands are overlapped by the dominating $3 \mathrm{p}$ band, all three bands appear separated at similar intensity in the spectrum recorded in the VIS/IR range. Transitions into $s$ and d states which are forbidden for free atoms become allowed in a liquid cluster, where the radial symmetry is broken and the states gain molecular character.

Previous energy resolved studies on He clusters by von Haeften et al (1997) revealed that the VIS/IR emission stems mainly from the relaxation via cascades of desorbed excited He atoms and excimer molecules. In general, VIS/IR emission is expected to be weak because the branching ratio between the direct decay to the ground state and the cascade relaxation to lower excited states scales with the third power of the emission energy. For He atoms and molecules which have been desorbed from He clusters, however, direct decay might be forbidden by the selection rule $\Delta l=1$. In such a case relaxation is possible only via cascades which leads to a significant enhancement of the VIS/IR luminescence with respect to the VUV fluorescence. For the case of excited He atoms at the cluster surface the mechanism is schematically explained in figure 1 .

Having identified the $3 \mathrm{~s}, 3 \mathrm{p}$ and $3 \mathrm{~d}$ states von Haeften et al (2001) varied systematically the cluster size and the isotopic constitution and analysed the size-dependent spectral shift of the $3 p$ band. It turned out that in He the key parameter controlling the energy of the electronically excited states appeared to be the density. Because of the loosely bound surface atoms the average density in He clusters depends on their size. When the cluster size becomes smaller the average density decreases likewise. Besides, the average density of He clusters depends considerably on the isotopic constitution since the ratio between the density of liquid ${ }^{3} \mathrm{He}$ and ${ }^{4} \mathrm{He}$ is $75 \%$. The spectra of small ${ }^{4} \mathrm{He}$ clusters and large ${ }^{3} \mathrm{He}$ clusters having identical average densities exhibited similar shifts. Moreover, the energy of the maximum of the $3 p$ band in the VIS/IR excitation spectrum correlated with the average density of the clusters. This behaviour is characteristic for atoms in a dense gas rather than for clusters where, as Jortner (1992) pointed out, usually confinement effects play a dominating role.

While the nature of the lower excited states in He clusters is conclusively explained by a model of perturbed atomic-type states much less is known about the higher states. In this region the absorption spectrum consists of diffuse broad bands overlapped by sharp peaks at the position of the atomic $n \mathrm{p}$ lines. With increasing cluster size the barycentre of these bands shift to higher energies (Joppien et al 1993). This trend is opposite to what is observed for heavier rare gas clusters. Their shifts increase when the size is reduced. It is therefore obvious that the nature of the excited states in He clusters cannot be explained by the concept of confined Wannier excitons which Wörmer et al (1996) found appropriate for heavier rare gas 


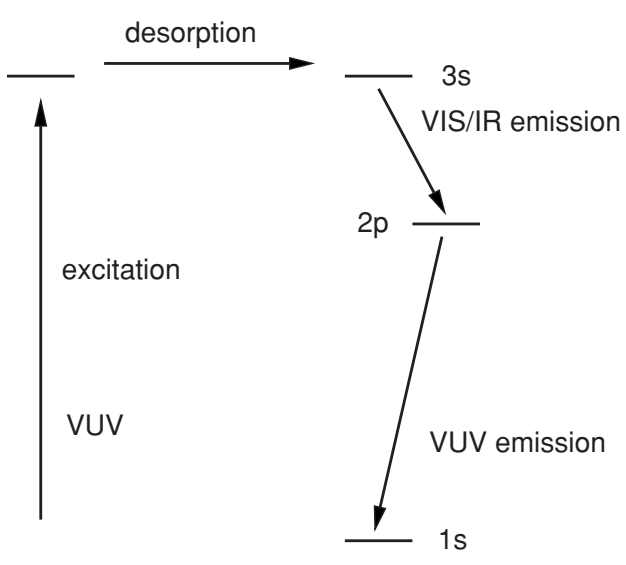

Figure 1. Scheme of the desorption process after excitation. First, surface atoms are excited into perturbed $3 \mathrm{~s}, 3 \mathrm{p}$, or $3 \mathrm{~d}$ states. After subsequent desorption from the cluster the He atoms reside in well-defined states. From $3 \mathrm{~s}$ or $3 \mathrm{~d}$ states they can decay only via cascades to the ground state. As a consequence VIS/IR radiation is enhanced with respect to the VUV fluorescence.

clusters. Atomic Rydberg-type states are also not expected in the interior of He clusters in this energy range. Since these states would tend to have large electron radii their wavefunctions would strongly overlap with those of the electrons of the 1s shells of the surrounding ground state He atoms. Because of the lack of space and because of the inhomogeneity such states cannot be discrete and orthogonal to each other.

On the other hand, Golov and Sekatskii (1993 and 1994) proposed several years ago the existence of Rydberg states in He clusters. They considered a positive charge which was delocalized over the cluster together with an electron which was orbiting outside the cluster on Rydberg trajectories around the cluster core. It is obvious that this type of Rydberg states can only exist in small He clusters. Lerner and Sokolov (1986 and 1989) showed that Rydberg atoms can be bound to the He surface by the attractive interaction between a $\mathrm{He}^{+}$ion with ground state He atoms and the interaction between the Rydberg electron with its image potential. According to the calculation atoms are bound for high principal quantum numbers $n$ and can float over the liquid helium (LHe) surface. Furthermore, Ancilotto et al (1995) predicted that only light atoms like $\mathrm{H}, \mathrm{He}$ and Li reside in a stable state above the LHe surface. The Rydberg levels are slightly perturbed depending on the polarizability $\epsilon$ of the liquid. The level shift is proportional to $(\epsilon-1) /(\epsilon+1)$.

In this paper we are concerned with the high energy part of the fluorescence excitation spectrum of He clusters which might be related to Rydberg states. For the analyses we consider spectra recorded in separate wavelength ranges or decay time intervals. The excitation spectrum of large He clusters exhibits broad, diffuse bands accompanied by sharp lines which are much weaker. The spectrum of small He clusters reveals sharp lines only. As we will show, the broad diffuse bands can be assigned to states connected to excitations in the interior part of the clusters. He clusters show no fluorescence above $\sim 25.7 \mathrm{eV}$ where the emission of free electrons becomes the dominating relaxation channel. The fluorescence cut-off lies more than $1 \mathrm{eV}$ above the ionization threshold energy of He atoms because the creation of free electrons in liquid He requires an energy of approximately $1 \mathrm{eV}$. The sharp peaks which show up in the spectra of large He clusters are assigned to excitations of surface atoms which were predicted by Lerner and Sokolov (1986 and 1989). For ${ }^{3} \mathrm{He}$ clusters the sharp peaks are stronger than for ${ }^{4} \mathrm{He}$, probably because of their lower polarizability and because they exhibit 


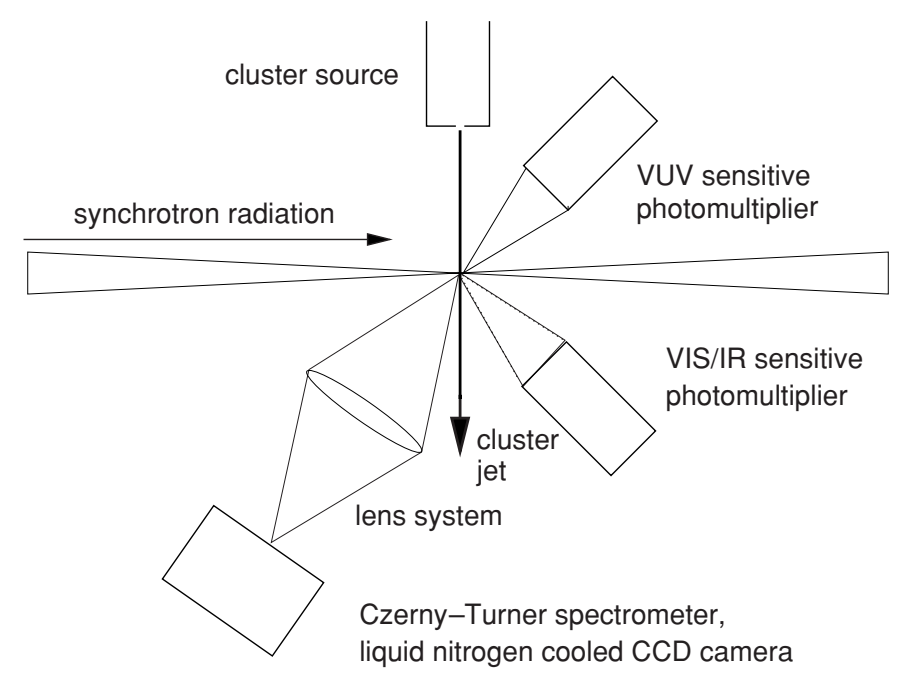

Figure 2. Schematics of the experimental set-up. The fluorescence of He clusters is recorded simultaneously by two photo multipliers which are sensitive in different wavelength ranges. The VUV fluorescence is either recorded by a XP2020, Valvo, covered with sodium salicylate, in an integral mode or, alternatively, by using a solar blind R2050, Hamamatsu multiplier in the range between 110 and $180 \mathrm{~nm}$. Luminescence in the VIS and near IR is recorded by using a Peltier-cooled R943-02, Hamamatsu.

more atoms in the surface region. The sharp lines which dominate the spectrum of small clusters likely indicate the existence of Rydberg states of the type which Golov and Sekatskii discussed in 1993. Energy resolved luminescence spectra taken at excitation energies close to the atomic ionization limit show the emission of He atoms with $n \geqslant 11$ and give further evidence that surface He atoms and highly vibrationally excited He molecules are excited into Rydberg states. Subsequently, the molecules dissociate, recombine or eventually desorb without further loss of energy and emit in vacuum.

\section{Experiment}

The experiments have been carried out at the experimental station $C L U L U$ at beam line $I$ at HASYLAB. A detailed description of the experimental set-up, however in an older version, is given by Karnbach et al (1993). Modifications of the experiment are described by Joppien et al (1993) and von Haeften et al (1997, 2001 and 2002). A schematic sketch is given in figure 2. He clusters are produced by supersonic expansion of cold He gas into vacuum through a small pinhole nozzle of $5 \mu \mathrm{m}$ in diameter. The purity of the He gas is $99.9999 \%$. Contaminations of the clusters by collisions and pick-up of residual gas molecules are extremely unlikely. Because of the background pressure being $1 \times 10^{-8}$ mbar and the short cluster flight distance of $2 \mathrm{~mm}$, such a process has a probability of distinctively less than $0.01 \%$ even for the largest clusters considered here. The average size of the clusters depends on the nozzle diameter $d_{0}$, the stagnation pressure $p_{0}$, the nozzle temperature $T_{0}$ and the isotopic composition of the He gas. While the nozzle diameter is fixed, the stagnation pressure is usually kept at 40 bar and the nozzle temperature is varied in the range from 5 to $35 \mathrm{~K}$ in order to adjust the average cluster size. The corresponding mean cluster sizes are derived from the results of a cross beam scattering experiment (Harms et al 1998 and 2001). This experiment allowed us to estimate the He cluster sizes with high accuracy. Moreover, we accounted for possible 
errors in measuring the nozzle temperature by comparing the threshold temperature for the formation of ${ }^{3} \mathrm{He}$ clusters with that found by Harms et al (1998). The cluster sizes which are reported here might deviate from the values of Harms et al by approximately $25 \%$ because of possible variations of the nozzle diameter between Harms' and our experimental set-up.

For the experiments with ${ }^{4} \mathrm{He}$ clusters also a $20 \mu \mathrm{m}$ nozzle was used. In this case $p_{0}$ was set to 12.5 bar. For a given nozzle temperature $T_{0}$ this combination revealed spectra that were similar to those which were recorded using the $5 \mu \mathrm{m}$ nozzle at $40 \mathrm{bar}$. The He cluster flux and the signal-to-noise ratio, however, could thus be improved.

In principle, the production of ${ }^{3} \mathrm{He}$ clusters is similar, however, the nozzle temperature $T_{0}$ lies in a lower regime $(7-10 \mathrm{~K})$. For $p_{0}$ we used 7 bar. The expensive ${ }^{3} \mathrm{He}$ gas was continuously recycled. During this recycling process the gas was compressed and carefully cleaned using zeolite traps which were cooled with liquid nitrogen. Otherwise, contaminations in the gas would likely block the nozzle. In order to evacuate the recycling system, also specially sealed roughing pumps were used. It has to be mentioned that the range in which the cluster size can be varied is limited for ${ }^{3} \mathrm{He}$. In contrast to ${ }^{4} \mathrm{He}$ condensation starts abruptly for ${ }^{3} \mathrm{He}$ clusters. With decreasing the nozzle temperature the size remains almost constant and rises then sharply to some 10000 atoms. As has been shown by Harms et al (2001) the smallest clusters are in the size range of several thousand ${ }^{3} \mathrm{He}$ atoms. Calculations by Pandharipande et al (1986), Stringari and Treiner (1987) and later Guirao et al (1991), Joyes et al (1991) and Barranco et al (1997) show that ${ }^{3} \mathrm{He}$ dimers and also small clusters with $N \sim 25$ are not bound. Therefore, the growth process of ${ }^{3} \mathrm{He}$ clusters must be different from that of ${ }^{4} \mathrm{He}$ clusters which could explain the absence of clusters smaller than a few thousand atoms.

Approximately $2 \mathrm{~mm}$ after the nozzle the He clusters were excited with monochromatized synchrotron radiation in the energy range between 19 and $26 \mathrm{eV}$ at a resolution of $18 \mathrm{meV}$. Upon excitation the He clusters decay via VUV fluorescence to the ground state. As has been found out by Karnbach et al (1993) non-radiative decay to the ground state is unlikely for rare gas clusters. Recording the VUV fluorescence yield is therefore appropriate to measure the photoabsorption spectrum. The VUV fluorescence yield was measured in the range between 30 and $300 \mathrm{~nm}$ by a XP 2020 photomultiplier tube (Valvo). A glass window placed in vacuum in front of the photomultiplier tube was covered with a layer of sodium salicylate which converted VUV photons into radiation to which the multiplier was sensitive. The spectral selectivity was tested by exciting a beam of $\mathrm{N}_{2}$ molecules. The VIS/IR emission of $\mathrm{N}_{2}^{+}$was not detected by this detector. Our apparatus allowed us also to record the luminescence in other distinct wavelength ranges. VIS/IR spectra were recorded by a Peltier cooled R 943 photomultiplier tube (Hamamatsu). This detector is sensitive from 170 to $850 \mathrm{~nm}$, but for the present study glass filters were used to limit the sensitivity to $\sim 300 \mathrm{~nm}$. The sensitivity ratio between the $\mathrm{R} 943$ and XP 2020 was measured using the $3 \mathrm{p}^{1} \mathrm{P}$ excitation of a He atomic beam. Since the branching ratio between the $3 \mathrm{p}^{1} \mathrm{P} \rightarrow 2 \mathrm{~s}^{1} \mathrm{~S}$ and the $3 \mathrm{p}^{1} \mathrm{P} \rightarrow 1 \mathrm{~s}^{1} \mathrm{~S}$ emissions is controlled by the well-known Einstein factors $A_{i, k}$ we could evaluate that the sensitivity of the R 943 was increased by 2.22 with respect to the XP 2020. Alternatively to the XP 2020 a solar blind R 2050 photomultiplier tube (Hamamatsu) mounted in vacuum chamber right above the focal point was used to record the fluorescence in the range between 115 and $180 \mathrm{~nm}$. Large He clusters emit a broad continuum radiation similar to liquid He (Surko et al 1970) and to He excimer molecules as Joppien et al (1993) reported. This continuum stems mainly from bound-free transitions between the lowest electronically excited He excimer state and the repulsive $\mathrm{He}-\mathrm{He}$ ground state potential at small interatomic distances $(1.1 \AA)$. A very small portion of the low energy tail of this continuum lies in the sensitivity range of the R 2050 photomultiplier. For all photomultipliers the fluorescence intensity was measured using the single photon counting technique. In order to account for the slowly decaying 


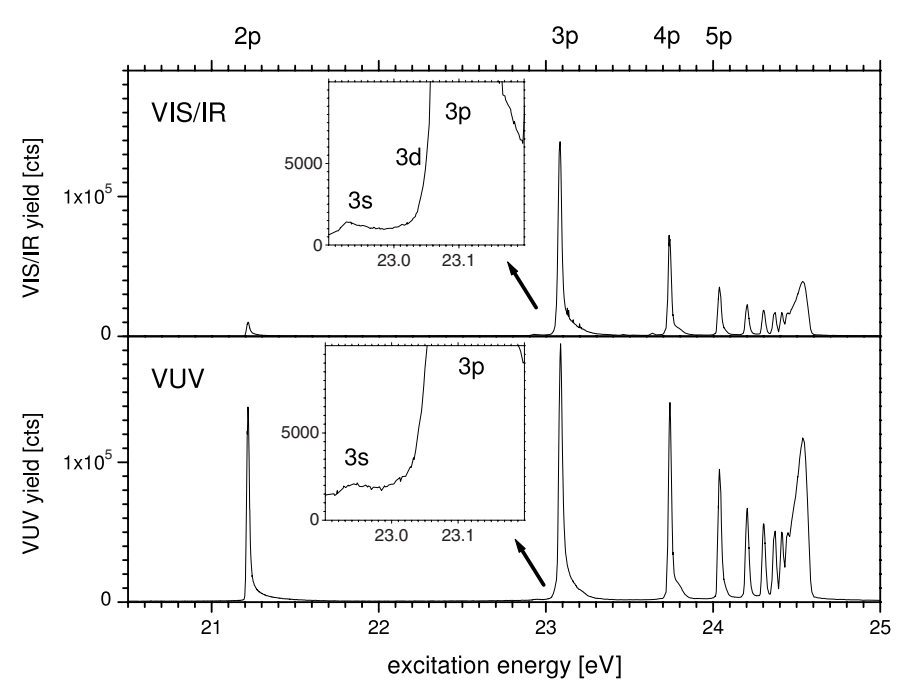

Figure 3. The spectra of $N=50{ }^{4} \mathrm{He}$ clusters are shown. The upper spectrum has been recorded using the VIS/IR sensitive detector, the lower one using the VUV sensitive detector. The enlarged insets show the weakly perturbed $3 \mathrm{~s}$ and $3 \mathrm{~d}$ features. The excitation into $3 \mathrm{~d}$ states can be identified as a shoulder of the low energy side of the $3 p$ line in the VIS/IR spectrum. Atomic energy levels are indicated on the top.

intensity of the synchrotron light the dwell time was controlled by the current of the storage ring. All excitation spectra were normalized to the spectral intensity distribution of the beam line.

Dispersed luminescence was recorded in the VIS/IR range by means of a $0.25 \mathrm{~m}$ CzernyTurner spectrometer equipped with a liquid nitrogen CCD camera. The spectrometer is sensitive in the range between 300 and $1100 \mathrm{~nm}$. At a slit width of $80 \mu \mathrm{m}$ the resolution is $2 \mathrm{~nm}$ when a grating width 150 grooves $\mathrm{mm}^{-1}$ is used. The exposure time was 5 or $10 \mathrm{~min}$. It is possible to group the pixels of the CCD chip. We have divided the illuminated area of the CCD chip into six stripes in that way that they allowed the simultaneous record of six spectra of comparable intensity. By comparing these six spectra with each other using a home-made program it was possible to identify pixel groups which revealed much higher intensities because of the accidental record of 'cosmic particle events'. In such a case the intensity of this pixel group is replaced by the average intensity of the five others. Finally, the six spectra are averaged. Using six stripes appeared to be the best compromise between a clean reduction of noise by cosmic particle events and a low read-out noise which increases linearly with the number of stripes.

Time correlated excitation spectra were recorded by making use of the pulsed nature of the synchrotron radiation which had a repetition rate of $5 \mathrm{MHz}$ at a pulse duration of $130 \mathrm{ps}$. The time between the detection of each of the fluorescence photons and the next excitation pulse was measured and the photons were counted only if this time was in the considered fluorescence lifetime interval. For the VUV yield the accuracy of time resolution was limited by the fluorescence lifetime of the sodium salicylate which is in the order of 15-20 ns.

\section{Results and discussion}

Figure 3 shows a spectrum of ${ }^{4} \mathrm{He}$ clusters comprising 50 atoms on average. The spectrum reveals mainly sharp lines which are located at the position of the atomic $n p$ levels. Each 
line is accompanied by weak wings to the high energy side of each line. The wings extend strongest to higher energies for the $n=2$ and 3 levels. For $n=4$ the shift becomes smaller whereas for $n=5$ and higher levels the wing is hardly to be seen. At the given resolution of our experimental apparatus the VUV fluorescence excitation spectrum of small He clusters $(N \leqslant 300)$ appears very similar to that of He atoms. This similarity is not observed for other rare gas clusters of the same size. Their spectra show broad bands even for $N \leqslant 10$ as Wörmer et al (1996) showed. An important difference between the spectra of He atoms and small clusters, however, is recognized when the excitation spectra which were recorded in the VIS/IR range are compared. In this spectrum the $2 p$ line shows up for small He clusters while it is absent for the atomic beam as has been shown by Möller et al (1999). For He atoms in the $2 \mathrm{p}$ state the only possible VIS/IR decay is the $2 \mathrm{p}^{1} \mathrm{P} \rightarrow 2 \mathrm{~s}^{1} \mathrm{~S}$ transition at $1088 \mathrm{~nm}$. Since this is out of the sensitivity range of the R 943 photomultiplier a VIS/IR line is absent. In small clusters, energy is transferred to He excimers which can emit in the VIS/IR, mainly via the $E^{1} \Pi_{\mathrm{g}} \rightarrow A^{1} \Sigma_{\mathrm{u}}^{+}$and $F^{1} \Delta_{\mathrm{u}},{ }^{1} \Pi_{\mathrm{u}},{ }^{1} \Sigma_{\mathrm{u}}^{+} \rightarrow B^{1} \Pi_{\mathrm{g}}$ transitions. Because of the presence of the $2 \mathrm{p}$ line in the VIS/IR excitation spectrum it is obvious that the features are due to He clusters and not to those He atoms which always accompany the cluster beam. An upper limit of the He atom fraction in the beam can be calculated by comparing the $3 p$ line intensities between the VIS/IR and the VUV excitation spectrum taking the increased sensitivity of the VIS/IR detector into account. According to the Einstein factor of emission the VIS/IR fraction of the atomic luminescence should be $2.3 \%$. Instead, the measured fraction is $35 \%$. Therefore, the upper limit for the fraction of atoms in the beam is $6.6 \%$. It seems reasonable to assume that the fraction of atoms is even lower than $6.6 \%$ because the efficiency of the energy transfer into states, which emit via VIS/IR cascades is likely $\leqslant 100 \%$. Another characteristic difference between the cluster and the atom spectrum is the sharp features at the $3 \mathrm{~s}$ and $3 \mathrm{~d}$ energies (inset in figure 3) since these transitions are forbidden for atoms. One reason for the ratio between the VIS/IR luminescence and the VUV fluorescence being 35\% is the desorption of excited He atoms in $3 \mathrm{~s}$ and $3 \mathrm{~d}$ states which is very specific for small clusters (see figure 1). Both the energy of the atomic $3 \mathrm{~d}$ and the $3 \mathrm{~s}$ state are lower than that of the $3 \mathrm{p}$ state. The interaction between ground state atoms which surround an excited $n \mathrm{p}$ He atom is repulsive due to the unfavourable overlap of both wavefunctions. Therefore, the energy of an excited level is shifted to higher energies, as well as the transition frequencies. The sharpness of the absorption lines is a consequence from the weak perturbation in small He clusters of $N=50$ which can easily be explained by the low density of the atoms. The average distance between the atoms of $4.2 \AA$ is considerably larger than the radius of the $2 p$ orbital which is $\sim 2.1 \AA$. Already the $n=4$ orbital exceeds the cluster radius which, according to Whaley (1994), is $7.8 \AA$ because of the quadratic dependence of the radius of the orbitals $r=a_{0} n^{2}$ on the principal quantum number $n$. Therefore, the states with higher principal quantum number could presumably be due to Rydberg states where the electron moves outside the cluster around a positive core, because the cluster diameter is smaller than the Rydberg radius. For most of the atoms in the cluster the repulsive overlap would be minimized which explains the absence of blue-shifted wings for $n \geqslant 6$ in the absorption spectrum of $N=50$ clusters.

The situation for large clusters is considered in figure 4 . Figure 4 shows time correlated excitation spectra recorded both in the VUV and in the VIS/IR range for clusters of $N=10000$ atoms. As we will show the technique of time correlated photon counting can significantly help to assign the spectral features. The VUV short lived fluorescence is measured within a $5 \mathrm{~ns}$ time interval $2.5 \mathrm{~ns}$ after the synchrotron light pulse. The delay of $2.5 \mathrm{~ns}$ is applied in order to reduce contributions from excitation stray light. Long lived fluorescence is recorded in a time interval between 40 and 140 ns. In addition, spectra recorded in the VIS/IR range were taken in the same time interval. For the short lived VIS/IR luminescence no extra delay 


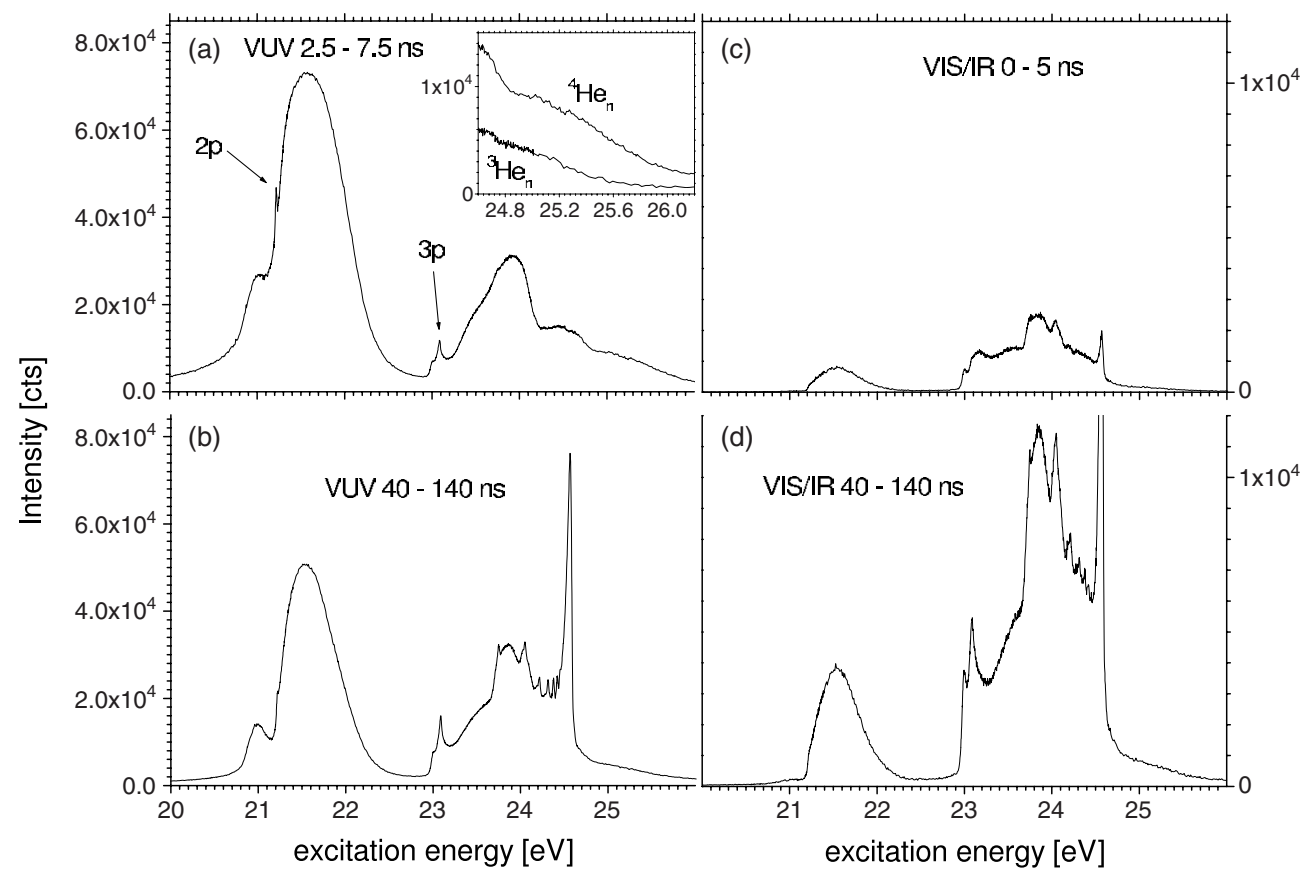

Figure 4. Time correlated excitation spectra of ${ }^{4} \mathrm{He}$ clusters with $N=10000$. The short lived and long lived VUV yield is shown in a and b, the short and long lived VIS/IR yield is shown in $\mathrm{c}$ and $\mathrm{d}$, respectively. The short lived features ( $\mathrm{a}$ and $\mathrm{c}$ ) are due to bulk, the long lived ( $\mathrm{b}$ and $\mathrm{d}$ ) are due to surface excitations. The inset in part a shows the short lived yield of ${ }^{3} \mathrm{He}$ and ${ }^{4} \mathrm{He}$ of the same size in the energy range above the atomic ionization threshold.

was needed, because there is no stray light in this wavelength range. We will show below that the spectral features in figures 4(a) and (c) are mainly due to absorption of bulk states while the features in figures 4(b) and (d) reflect mainly surface states. The spectrum in figure 4(a) shows with the exception of the sharp features at the $2 p$ and $3 p$ level mainly broad bands. The two lowest bands have been labelled ' $A$ ' and ' $B$ '. The bands at higher energy are difficult to assign because whether they appear or not depends strongly on the sensitivity range of the detector and also on the selected time interval. It is remarkable that the features extend above the ionization limit of He atoms at $24.56 \mathrm{eV}$. For comparison also a part of the corresponding spectrum of ${ }^{3} \mathrm{He}$ clusters of which the intensity has been normalized to the integrated ${ }^{4} \mathrm{He}$ spectrum is shown. The shift of the cut-off of the short-lived VUV-fluorescence is $1.35 \mathrm{eV}$ for ${ }^{4} \mathrm{He}$ and $0.95 \mathrm{eV}$ for ${ }^{3} \mathrm{He}$ clusters, respectively. The ${ }^{3} \mathrm{He}$ short lived VUV spectrum does not extend that much above the ionization threshold. A shifted cut-off energy for the VUV fluorescence is certainly due to the barrier for the injection of free electrons into the conduction band of liquid helium as it has been discussed by Buchenau et al (1991) who predicted a barrier of $1.1 \mathrm{eV}$ for ${ }^{4} \mathrm{He}$ clusters. Above this barrier a drop of fluorescence is expected because the electrons move through the cluster and eventually escape. Below $25.7 \mathrm{eV}$ recombination is possible. Recombination is likely to be followed by fast non-radiative decay. Subsequently, the clusters emit in the VUV from lower states, for instance the $2 p$ atomic state, which has a lifetime of $0.5 \mathrm{~ns}$ (Radzig and Smirnov 1985). Therefore, the bands above the ionization limit are detected preferentially when the short lived fluorescence is recorded. 


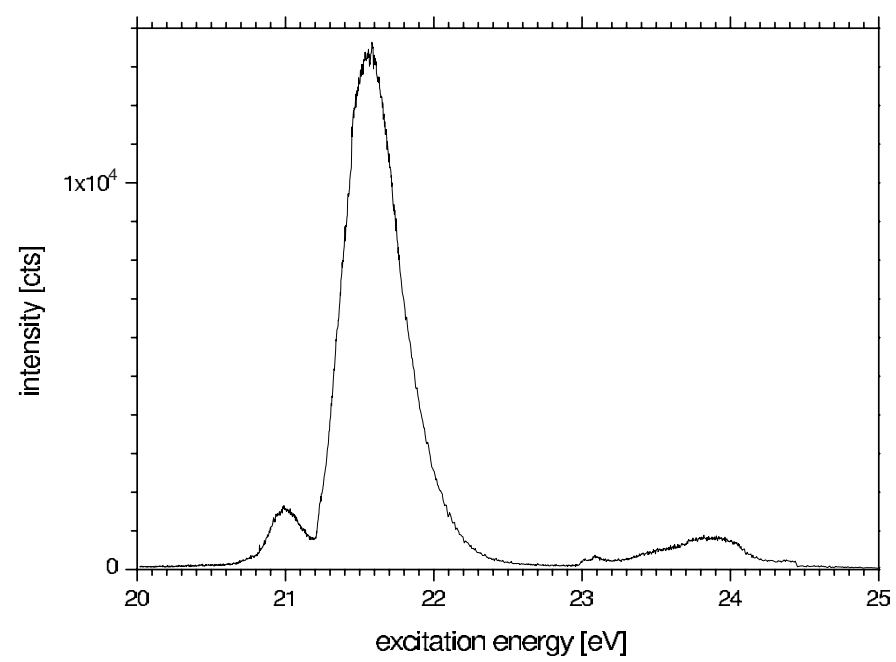

Figure 5. Excitation spectrum recorded using the solar blind photomultiplier. The solar blind detector is insensitive to the radiative emission of He atoms. The missing sharp lines in this spectrum indicate that they are specifically associated with the desorption of excited He atoms from the cluster. Since this relaxation channel is presumably favoured at the surface the absence or presence of sharp lines can be taken as an indicator for the location of excitations in a cluster.

The barrier shifts which we have found in this work agree qualitatively with those reported by Woolf and Rayfield (1965) for bulk liquid ${ }^{4} \mathrm{He}$ and Schoepe and Rayfield (1973) for ${ }^{3} \mathrm{He}$. The height of the barrier depends in the first approximation linearly on the number density. Broomall et al (1976) have measured the energy for electron injection into liquid ${ }^{3} \mathrm{He}$ and ${ }^{4} \mathrm{He}$ over a large range of densities. Their values for saturated vapour pressure of $0.8 \mathrm{eV}$ for ${ }^{3} \mathrm{He}$ and $1.25 \mathrm{eV}$ for ${ }^{4} \mathrm{He}$ agree quite well with our values found for clusters comprising $N=$ 10000 atoms.

The short life time of the atomic $2 \mathrm{p}$ state is responsible for the sharp $2 \mathrm{p}$ line in the spectrum in figure 4(a). The sharp peak is either due to He atoms in the background gas or loosely bound atoms at the outermost cluster surface where the perturbation is negligible. The $3 p$ line also appears in the long lived spectrum although the life time of the atomic $3 p$ state is short. The analyses of energy resolved luminescence spectra of He clusters of various sizes reveal that exceptionally after excitation of the sharp $3 p$ features the energy is transferred strongly from surface atoms into molecular He states. Such an energy transfer has been reported by Wellenstein and Robertson Wellenstein and Robertson (1972) for He in a gas.

Because of their diffuseness the broad bands are assigned to excitations in the interior of the cluster, where the magnitude of perturbation and inhomogeneity is large. The main reason for the increased sensitivity of the short lived features to the bulk states is that the density of states is certainly higher in the bulk than at the surface. Thus, non-radiative decay is expected to be faster. We believe therefore, that the broad features in figure 4(a) represent bulk excitations which decay via non-radiative mechanism to the short lived lower states, such like perturbed $2 \mathrm{p}$ states.

Our assignment is supported by the spectrum of large clusters $(N=10000)$ which has been recorded using the solar blind multiplier (figure 5). As explained above, the multiplier is sensitive only to a small portion of the long wavelength emission band of $\mathrm{He}_{2}^{*}$. In other words, the solar blind multiplier is completely insensitive to the VUV emissions of those 


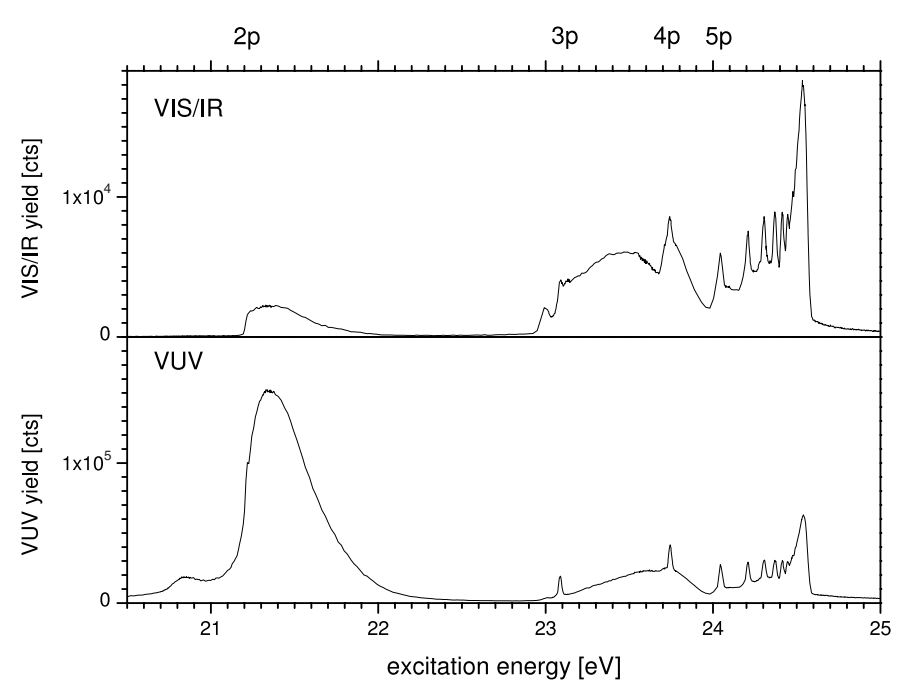

Figure 6. VIS/IR and VUV excitation spectra of $N=10000{ }^{3} \mathrm{He}$ clusters.

excited He atoms which are desorbed from the cluster. Therefore, we believe, that the solar blind spectrum is specific for emissions in the bulk region. It is reasonable to assume that the desorption of excited atoms is more likely for excitations at or close to the surface. The solar blind spectrum shows broad bands only. Sharp features are completely absent. This gives further evidence that the broad features are due to excitations in the interior.

It is more likely that the sharp features are due excitations at the surface. Here, the perturbation is less strong because of the lower interatomic separation and the lower coordination number. As can be seen in figure 4(b) the sharp features occur specifically in the long lived region. This is explained by immediate desorption of excited He atoms and molecules from surface sites which have long radiative lifetimes. With the exception of the sharp $3 p$ line the energy transfer into the interior region of the clusters is assumed to be weak. In many cases the desorbed species decay via cascades because direct decay to the ground state is forbidden. Generally, the radiative life time for the VIS/IR decay in the vacuum is larger than $10 \mathrm{~ns}$. Especially atomic Rydberg states are long lived.

This scenario is supported by the excitation spectra recorded in the VIS/IR range which are shown in figures 4(c) and (d). While the short lived spectrum is dominated by broad features the long lived spectrum displays sharp lines. Moreover, the intensity ratio between the short lived and the long lived spectra is about $1: 5$ while for the spectra which were taken in the VUV the ratio is $1: 0.6$. This intensity ratio reflects that the VIS/IR spectrum which is specifically sensitive to the desorption of excited species is, as we believe, rather characteristic for excitations at or close to the surface than for the bulk.

It has to be mentioned that the width of the sharp lines both in figures 4(b) and (d) is broader than the lines of an atomic beam $(\sim 18 \mathrm{meV})$. This is clearly visible, especially for the case of the $5 \mathrm{p}$ line which has a width of $68 \mathrm{meV}$. We believe that the sharp lines could likely be due to excitations of surface atoms from the ground state into Rydberg states. As Lerner and Sokolov (1989) demonstrated such Rydberg atoms become easier stabilized with increasing quantum number $n$. The line broadening reflects the perturbation of the Rydberg atoms.

Spectra of large ${ }^{3} \mathrm{He}$ clusters comprising 10000 atoms are shown in figure 6. Compared to the spectra of ${ }^{4} \mathrm{He}$ clusters of the same size the intensity of the sharp lines is stronger, 


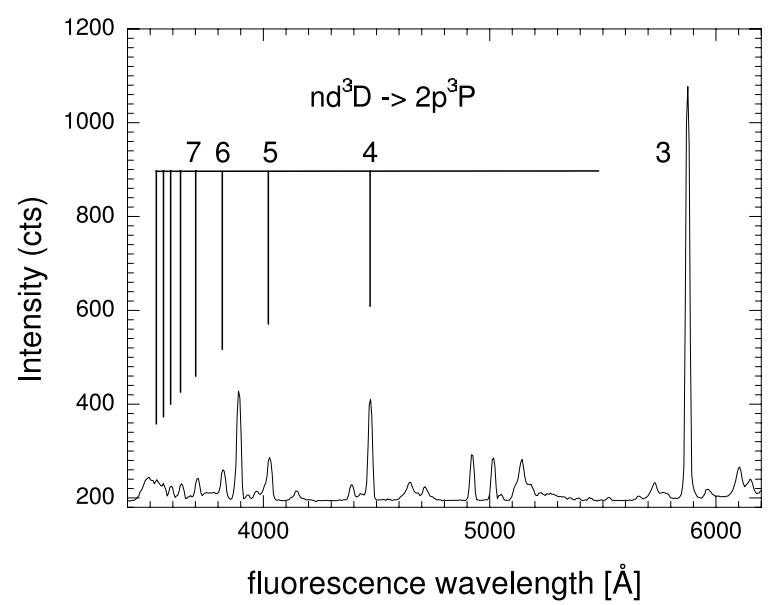

Figure 7. The emission spectrum displays the emission lines of the atomic Rydberg series $n \mathrm{~d}^{3} \mathrm{D} \rightarrow 2 \mathrm{p}^{3} \mathrm{P}$. The excitation energy was $24.5 \mathrm{eV}$.

especially in the VIS/IR spectrum. It is also clearly visible that the lines are broadened with respect to atomic lines. The magnitude of the line width is strongest for the $4 \mathrm{p}$ level (190 meV) and decreases with increasing principal quantum number $n$. This agrees with the predictions by Lerner and Sokolov (1986 and 1989) who pointed out that the Rydberg states of atoms on the surface are perturbed by the interaction with the surface. The magnitude of the perturbation, however, becomes smaller with increasing $n$. For excitations in the interior region of the clusters the dependence of the magnitude of perturbation on $n$ is opposite which underlines our interpretation.

At least Rydberg states on the surface of large He droplets are also involved in the relaxation process in He clusters. The energy resolved luminescence spectrum as shown in figure 7 shows the emission features of $N=10000{ }^{3} \mathrm{He}$ clusters in the VIS/IR range after irradiating the clusters with $24.5 \mathrm{eV}$ photons which is the strong resonance at the atomic ionization threshold. Among other molecular and atomic transitions the dominating $n \mathrm{~d}^{3} \mathrm{D} \rightarrow$ $2 \mathrm{p}^{3} \mathrm{P}$ Rydberg series is resolved up to $n=11$.

The atomic levels from which the emission occurs must be populated by non-radiative decay processes in the clusters. As we will show in the following these processes involve high vibrational levels of high molecular Rydberg states or the $\mathrm{X}^{2} \Sigma_{\mathrm{u}}^{+}$ground state of the $\mathrm{He}_{2}^{+}$ion. A schematic potential energy curve diagram is displayed in figure 8. At excitation energies around $24.5 \mathrm{eV}$ resonant excitation into molecular Rydberg states is favourable because of the following reasons: with increasing average interatomic separations in He clusters the FranckCondon factors of transition between the ground state and molecular Rydberg states with high vibrational quantum number $v$ close to the dissociation limit are fairly large. In particular, this means that for the loosely bound atoms at the surface the Franck-Condon factor is larger than for atoms in the interior. In ${ }^{3} \mathrm{He}$ clusters this effect is even stronger because the average distance between the atoms at the surface is smaller than in ${ }^{4} \mathrm{He}$. Moreover, resonant transitions into molecular Rydberg states should be favoured because the density of both electronic and vibrational states becomes larger with increasing the excitation energy towards the atomic ionization limit. In fact, a high transition probability for these states is recognized in the excitation spectrum in figure 6 which displays intense, sharp peaks at the atomic ionization threshold. 


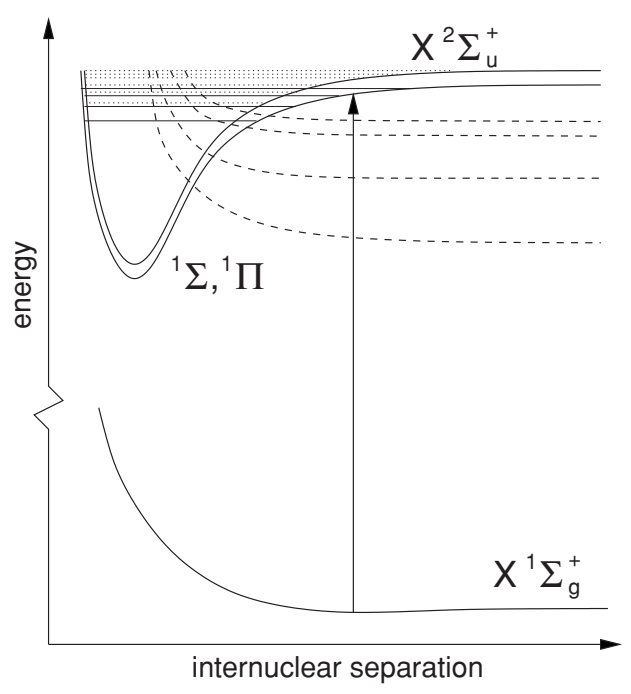

Figure 8. Scheme of the potential energy curves of $\mathrm{He}_{2}$ which refers to the emission spectrum in figure 7. The upper bound potential energy curve is due to the $\mathrm{X}^{2} \Sigma_{\mathrm{u}}^{+}$state, the lower represents the potential curves of the converging molecular Rydberg states. The lowest potential curve is due to the $\mathrm{X}^{1} \Sigma_{\mathrm{g}}^{+}$ground state of $\mathrm{He}_{2}$. The molecular Rydberg levels are solid, the ionic dotted and the dissociative curves are dashed. The excitation which is marked with an arrow occurs at large internuclear separation. The Franck-Condon overlap is large for excitations into highly vibrational molecular levels. Also, there is a high density of states close to the atomic ionization limit.

The origin of the emission spectrum in figure 7 is explained as follows: after excitation the energy is transferred into high vibrational $\mathrm{X}^{2} \Sigma_{\mathrm{u}}^{+}$states and the Rydberg molecule becomes ionized:

$$
\mathrm{He}_{2}^{*} \rightarrow \mathrm{He}_{2}^{+}+\mathrm{e}^{-}
$$

Because of crossing with dissociative potential curves the $\mathrm{He}_{2}^{+}$can recombine and dissociate. Recombination is also expected from the fact that the interaction of a free electron with liquid $\mathrm{He}$ is repulsive. The electron will therefore be scattered strongly at ground-state atoms in the cluster and cannot escape from the ion. This process was considered by Peterka et al (2003) in order to explain the zero kinetic energy of photoelectrons emitted from $\mathrm{He}$ clusters and also by von Haeften et al (1997) who explained emission from triplet states. Dissociative recombination of $\mathrm{He}$ ions

$$
\mathrm{He}_{2}^{+}+\mathrm{e}^{-} \rightarrow \mathrm{He}^{*}+\mathrm{He}
$$

which has been reported by Wellenstein and Robertson Wellenstein and Robertson (1972) is a well-known process in the gas phase.

The intensity distribution of the $n \mathrm{~d}^{3} \mathrm{D} \rightarrow 2 \mathrm{p}^{3} \mathrm{P}$ lines shows that emissions from lower levels are favoured. This might be due to the fact that the excited atoms gain more kinetic energy when the crossing occurs at lower curves. The higher curves are almost horizontal at the curve crossings. Therefore we believe, that at these states multiple associative ionization followed by dissociative recombination takes place.

Each time dissociative recombination takes place the electron spin can couple either to triplet or to singlet states, whereas triplet population should have a three times higher probability. Therefore, multiple associative ionization and dissociative recombination eliminate the population of high singlet levels which is at least observed in our study. 
In conclusion, we have studied the character of the higher electronically excited states in He clusters using time correlated fluorescence spectroscopy in specific wavelength intervals. The highly excited states of small He clusters might have Rydberg character because the Rydberg radius is considerably larger than the cluster radius. Because of the negative electron affinity of liquid He the electron tends to move outside the cluster. The Rydberg levels are only weakly perturbed because of the low dielectric constant of liquid He. Comparing the short with long lived spectra reveals features of large clusters which are due to excitations in the interior region. The features extend about $1.35 \mathrm{eV}\left(0.95 \mathrm{eV}\right.$ for $\left.{ }^{3} \mathrm{He}_{n}\right)$ above the ionization limit of He atoms which is in agreement with the findings for the penetration barrier of an electron into liquid ${ }^{4} \mathrm{He}$ and ${ }^{3} \mathrm{He}$. On the other hand, long lived features are due to excitations at the surface. The corresponding spectra show sharp lines at the atomic $n$ p levels. The sharp lines are likely to be due to atomic or molecular Rydberg states at the surface. Energy resolved luminescence spectra reveal VIS/IR emissions of Rydberg atoms. With increasing principal quantum number $n$ these emissions tend to arise from triplet levels. The analyses show that in a first step high vibrational quanta of molecular Rydberg states are populated when loosely bound atoms at the surface are excited. Atomic triplet levels are populated by sequential associative ionization and dissociative recombination.

\section{Acknowledgments}

The authors acknowledge valuable discussions with Karin Fink and Volker Staemmler, both at the Department of Theoretical Chemistry, Ruhr-Universität Bochum, Germany.

\section{References}

Ancilotto F, Cole M W, DeToffol G, Lerner P B and Toigo F 1995 J. Low Temp. Phys. 101 325-30 Arms D A, Simmons R O, Schwoerer-Böhning M, Macrander A T and Graber T J 2001 Phys. Rev. Lett. 87156402 Barranco M, Navarro J and Poves A 1997 Phys. Rev. Lett. 78 4729-32 Broomall J R, Johnson W D and Onn D 1976 Phys. Rev. B 14 2819-25 Buchenau H, Toennies J P and Northby J A 1991 J. Chem. Phys. 11 8134-48 Golov A and Sekatskii S 1993 Z. Phys. D 27 349-55

Golov A and Sekatskii S 1994 Physica B: Condens. Matter 194-196 555-6

Guirao A, Pi M and Barranco M 1991 Z. Phys. D 21 185-8

Harms J, Toennies J P, Barranco M and Pi Marti 2001 Phys. Rev. B 1884513

Harms J, Toennies J P and Dalfovo F 1998 Phys. Rev. B 58 3341-50

Joppien M, Karnbach R and Möller T 1993 Phys. Rev. Lett. 71 2654-7

Joppien M, Müller R and Möller T 1993 Z. Phys. D 26 175-7

Jortner J 1992 Z. Phys. D 24 247-75

Joyes P, Tarento R J and Van de Walle J 1991 Z. Phys. D 20 273-6

Karnbach R, Joppien M, Stapelfeldt J, Wörmer J and Möller T 1993 Rev. Sci. Instrum. $642838-49$

Lerner P B and Sokolov I M 1986 JETP Lett. 44 644-7

Lerner P B and Sokolov I M 1989 Z. Phys. D 14 173-7

Möller T, von Haeften K, Laarmann T and von Pietrowski R 1999 Eur. Phys. J. D 9 5-9

Pandharipande V R, Pieper S C and Wiringa R B 1986 Phys. Rev. B 34 4571-82

Peterka D S, Lindinger A, Poisson L, Ahmed M and Neumark D M 2003 Phys. Rev. Lett. 91043401

Radzig A A and Smirnov B M 1985 Reference Data on Atoms, Molecules and Ions (Berlin: Springer) p 232

Schoepe W and Rayfield G W 1973 Phys. Rev. A 7 2111-21

Stringari S and Treiner J 1987 J. Chem. Phys. 87 5021-7

Surko C M, Dick G J, Reif F and Walker W C 1969 Phys. Rev. Lett. 23 842-6

Surko C M, Packard R E, Dick G J and Reif F 1970 Phys. Rev. Lett. 24 657-9

von Haeften K, de Castro A R B, Joppien M, Moussavizadeh L, von Pietrowski R and Möller T 1997 Phys. Rev. Lett. 78 4371-4 
von Haeften K, Laarmann T, Wabnitz H and Möller T 2001 Phys. Rev. Lett. 87153403 von Haeften K, Laarmann T, Wabnitz H and Möller T 2002 Phys. Rev. Lett. 88233401 Wellenstein H F and Robertson W W 1972 J. Chem. Phys. 56 1077-82

Whaley B K 1994 Int. Rev. Phys. Chem. 13 41-84

Woolf A M and Rayfield G W 1965 Phys. Rev. Lett. 15 235-7

Wörmer J, Karnbach R, Joppien M and Möller T 1996 J. Chem. Phys. 104 8269-78 